\title{
Nonlinear evolution of the momentum dependent condensates in strong interaction: the "pseudoscalar laser"
}

\author{
Adrian Dumitru $^{a}$ and Ove Scavenius ${ }^{b}$ \\ ${ }^{a}$ Physics Department, Columbia University, 538W 120th Street, New York, NY 10027, USA \\ ${ }^{b}$ The Niels Bohr Institute, Blegdamsvej 17, DK-2100 Copenhagen Ø, Denmark
}

(October 25, 2018)

\begin{abstract}
We discuss the relaxation of the scalar and pseudoscalar condensates after a rapid quench from an initial state with fluctuations. If we include not only the zero-mode but also higher modes of the condensates in the classical evolution, we observe parametric amplification of those "hard" modes. Thus, they couple nonlinearly to the "soft" modes. As a consequence, domains of coherent $\pi$-field emerge long after the initial spinodal decomposition. The momentum-space distribution of pions emerging from the decay of that momentumdependent condensate is discussed.
\end{abstract}

The exciting speculation that the dynamics of spontaneous breaking of chiral symmetry could reorient the condensates in QCD as compared to the physical vacuum has stimulated many studies, see e.g. [1] [7], and [8] for reviews. However, for the chiral symmetry breaking process in strong interaction the spinodal decomposition after a rapid "quench" does not yield a large correlation length [9]. Accordingly, a simulation [4] of the classical equations of motion of the linear $\sigma$-model on a 3d-lattice with spacing $a=1 \mathrm{fm}$ confirmed that in the strongcoupling case no large domains form in which the $\pi$-field were essentially uniform, see also [5].

We shall confirm that result but also argue that if the cutoff for the Fourier modes of the classical order parameter of chiral symmetry is pushed to larger values by some physical mechanism, e.g. parametric resonance [10 12], domains much bigger than the Compton wavelength of the pion can form over time-scales $\gg 1 \mathrm{fm}$. Thus, the "pseudoscalar laser" is a non-linear phenomenon developing long after spinodal decomposition.

Our rather simple idea is that parametric resonance leads to large occupation numbers of "hard" modes of the scalar and pseudoscalar condensates, which then couple non-linearly to the "soft" modes. Thus, the same mechanism that is responsible for the explosive heating of the universe after inflation [10] (see also [13] were the inflaton decay was studied via a simulation on the lattice, similar to our studies here) leads to the formation of domains where the pseudoscalar condensate exhibits coherent oscillations with big amplitude around its vacuum value. Those domains could be produced in the laboratory in collisions of protons [1] or heavier nuclei [2] at high energies.
As an effective theory of the chiral symmetry breaking dynamics [14] we apply the linear $\sigma$-model [15]

$$
\begin{aligned}
\mathcal{L} & =\bar{q}\left[i \gamma^{\mu} \partial_{\mu}-g\left(\sigma+i \gamma_{5} \vec{\tau} \cdot \vec{\pi}\right)\right] q \\
& +\frac{1}{2}\left(\partial_{\mu} \sigma \partial^{\mu} \sigma+\partial_{\mu} \vec{\pi} \partial^{\mu} \vec{\pi}\right)-U(\sigma, \vec{\pi}) .
\end{aligned}
$$

The potential exhibiting the spontaneously broken symmetry is

$$
U(\sigma, \vec{\pi})=\frac{\lambda^{2}}{4}\left(\sigma^{2}+\vec{\pi}^{2}-v^{2}\right)^{2}-H \sigma .
$$

Here $q$ is the constituent-quark field $q=(u, d)$. The scalar field $\sigma$ and the pseudoscalar field $\vec{\pi}=\left(\pi_{1}, \pi_{2}, \pi_{3}\right)$ together form a chiral field $\Phi=(\sigma, \vec{\pi})$. The parameters of the Lagrangian are usually chosen such that the chiral $S U_{L}(2) \otimes S U_{R}(2)$ symmetry is spontaneously broken in the vacuum and the expectation values of the condensates are $\langle\sigma\rangle=f_{\pi}$ and $\langle\vec{\pi}\rangle=0$, where $f_{\pi}=93 \mathrm{MeV}$ is the pion decay constant. The explicit symmetry breaking term is determined by the PCAC relation which gives $H=f_{\pi} m_{\pi}^{2}$, where $m_{\pi}=138 \mathrm{MeV}$ is the pion mass. Then one finds $v^{2}=f_{\pi}^{2}-m_{\pi}^{2} / \lambda^{2}$. The $\sigma$-mass, $m_{\sigma}^{2}=2 \lambda^{2} f_{\pi}^{2}+m_{\pi}^{2}$, which we set to $600 \mathrm{MeV}$, yields $\lambda^{2} \approx 20$. For the moment we focus exclusively on the dynamics of the chiral condensates, i.e. we set $g=0$. We return to the case $g \neq 0$ at the end.

We shall discuss the evolution of the system in the potential 0.2 , starting from a chirally symmetric initial state, $\langle\Phi\rangle=0$. Such an initial state could be reached thanks to the slow dynamics of the longwavelength modes [3 [5,9]; or, in the case $g>0$, due to a possible first-order symmetry breaking phase transition which "traps" the expectation value of the fields in the local minimum around $\langle\Phi\rangle=0 \sqrt{6}$. We include fluctuations in the initial state by distributing the fields as Gaussian random variables with [3, 1 ,

$$
\left\langle\Phi_{a}^{2}\right\rangle=v^{2} / 16 \quad, \quad\left\langle\dot{\Phi}_{a}^{2}\right\rangle=v^{2} / 4 \mathrm{fm}^{2} \quad .
$$

Since $\langle\Phi\rangle=0$, the system is in an unstable condition for the potential at hand, eq. (0.2). The fields "roll down" towards the vacuum. In analogy to condensed matter physics this process is called spinodal decomposition [4, 9]. 


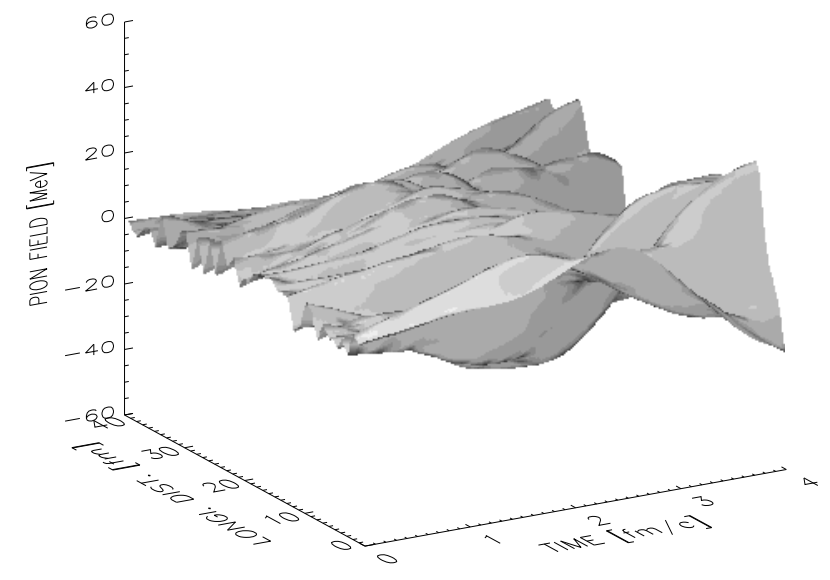

FIG. 1. Time evolution of the $\pi$-field in the longitudinal space-direction, and isospin-0 direction, averaged over the transverse directions, time $0-4 \mathrm{fm}$ (practically independent of lattice spacing as long as $0.1 \mathrm{fm} \leq a \leq 1 \mathrm{fm}$ ).

We first show numerical solutions of the classical equations of motion obtained from (0.1) on a lattice of physical size $10 \mathrm{fm} \times 10 \mathrm{fm}$ in "transverse" directions, and $40 \mathrm{fm}$ in longitudinal direction. (Throughout the manuscript Minkowski-metric has been employed). We have integrated the equations using a second-order leap-frog algorithm, ensuring that energy and momentum were conserved to within $0.1 \%$. We performed simulations with lattice spacing $a=1 \mathrm{fm}$ as well as $a=0.2 \mathrm{fm}$. In the latter case, a coarse-graining procedure was applied to the initial configuration to obtain a correlation length of $1 \mathrm{fm}$; this was done without artificially cooling the fluctuations $(0.3)$.

Fig. 1 depicts the $\pi$-field averaged over the transverse plane, showing the evolution within the first $4 \mathrm{fm}$. One observes that the field amplitude within each individual small initial "domain" gets amplified, exactly as it should be [3]. However, the wavelengths of the $\pi$-field oscillations more or less correspond to those of a free pion. Even for the rather small initial fluctuations (0.3) one can hardly observe big domains emerging during the initial "roll-down" [9,5], the less the larger the fluctuations at $t=0$. Also, we have found that the amplitude of the $\pi$-field after "roll-down" are almost independent of $a$. This is no longer true for the long-time evolution to be discussed next.

For large lattice spacing no big domains can be observed even at much later times, $t=80-100 \mathrm{fm}$, as shown in Fig. 2. The field is basically exhibiting only small and short-range oscillations.

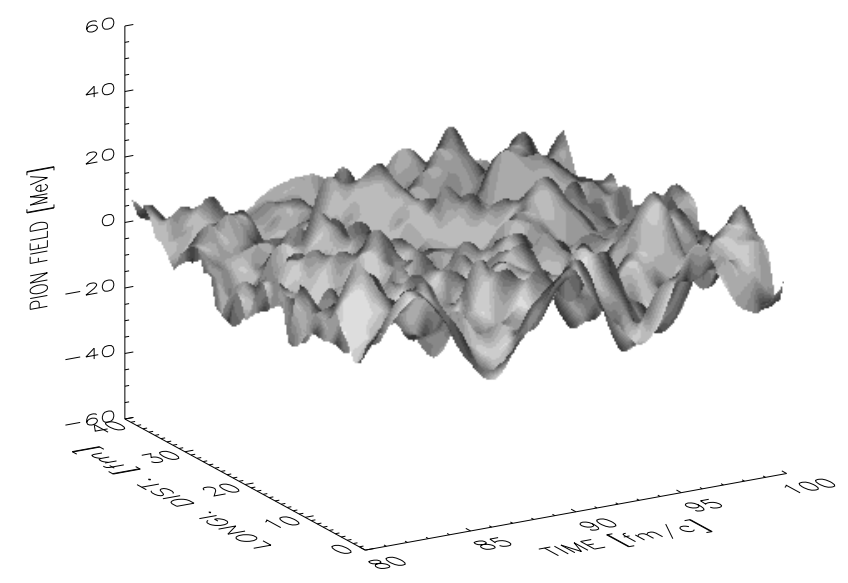

FIG. 2. As Fig. 1 but at time $80-100 \mathrm{fm}$ (lattice spacing $a=1 \mathrm{fm})$.

However, for $a=0.2 \mathrm{fm}$, one can observe long-range oscillations with a much bigger amplitude, clearly exhibiting the strong amplification of the long-wavelength modes and, correspondingly, big domains (Fig. 3). Moreover, this observation remains true even for larger initial fluctuations.

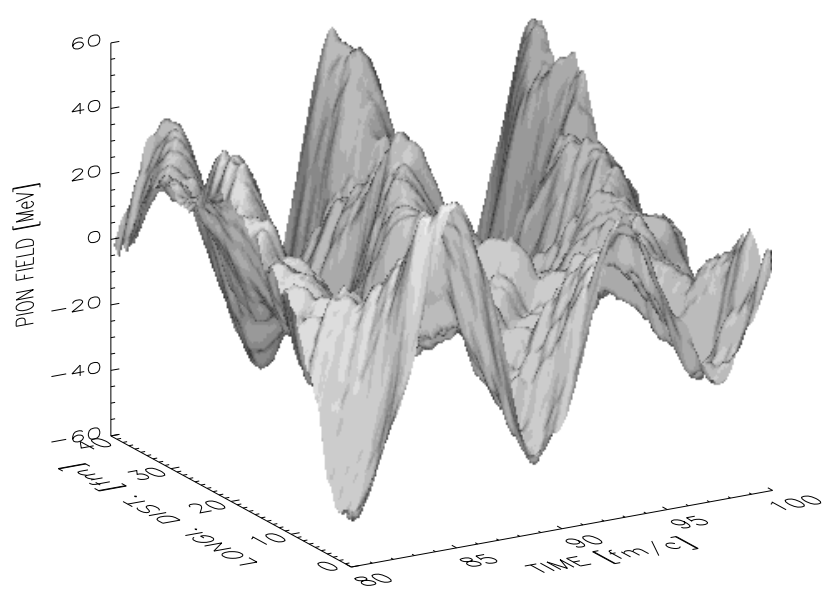

FIG. 3. As Fig. 目 but at time $80-100 \mathrm{fm}$ (lattice spacing $a=0.2 \mathrm{fm})$.

In Fig. 1 we show the momentum-space distribution of $\pi$-particles in the condensate (the distribution observed if the field decayed into particles at that moment) obtained from the spacial Fourier transform of $\pi(t, \vec{x})$. For simplicity, when computing $d N / d^{3} k$ we assumed the Hartree dispersion relation $\Omega^{2}=k^{2}+\lambda^{2}\left(\left\langle\sigma^{2}\right\rangle+5\left\langle\pi_{0}^{2}\right\rangle / 3-v^{2}\right)$. (The even more simple-minded application of the freepion dispersion relation gave practically the same result.) 


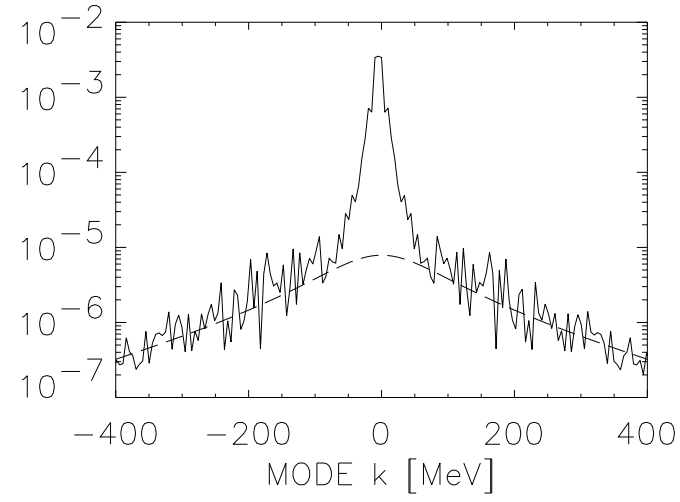

FIG. 4. Distribution of pions of given isospin 0 in momentum space, $d N / d^{3} k$ (lattice spacing $a=0.2 \mathrm{fm}$, time $t=100 \mathrm{fm}$ ). The broken line depicts a statistical (Bose) distribution.

The result has been averaged over several initial field configurations, chosen randomly according to (D.3). One observes a very sharp rise below $k \sim 70 \mathrm{MeV}$, proving the spacial homogeneity of the pseudoscalar condensate at $t=100 \mathrm{fm}$. Note that a statistical (Bose-Einstein) distribution does not show such a "rise", i.e. local maximum of the second derivative, at non-vanishing $k$. (In Fig. 4, the statistical distribution is for a temperature $T=160 \mathrm{MeV}$ and a chemical potential $\mu_{\pi}=100 \mathrm{MeV}$.)

To understand the results of the simulation we analyze the effect of the $\sigma$-field oscillations on the Fourier modes of the $\pi$-field in a more schematic model. We employ the linearized classical equation of motion for the pion field, neglecting also explicit symmetry breaking $\left(m_{\pi}=0\right)$. Furthermore, we assume that the sigma-field makes harmonic oscillations with amplitude $\sigma_{0}$ in the minimum of the potential, $\sigma=\sigma_{0} \sin \left(m_{\sigma} t\right)$. After Fourier transformation, the evolution of the $\pi$-field modes becomes (see also [10])

$$
\ddot{\pi}_{k}+\left\{k^{2}+\lambda^{2} \sigma(\sigma+2 v)\right\} \pi_{k}=0 .
$$

The expression in curly brackets gives the frequency $\Omega_{k}^{2}$ of the $\pi$-field oscillations. As initial condition for the $\pi$-field we assume $\pi_{k}(t=0)=1 / \sqrt{2 \Omega_{k}}, \dot{\pi}_{k}(t=0)=\sqrt{\Omega_{k} / 2}$. The solution of this equation can be found numerically without great difficulties (we used the routine "odeint" from [16], modified to work with double precision, and required accuracy $10^{-9}$ ).

Fig. 5 shows the occupation number

$$
n_{k}^{\pi}=\frac{\Omega_{k}}{2}\left(\frac{\left|\dot{\pi}_{k}\right|^{2}}{\Omega_{k}^{2}}+\left|\pi_{k}\right|^{2}\right)-\frac{1}{2}
$$

of the $\pi$-field modes for three different $\sigma$-field amplitudes. (For $\sigma_{0} \ll v$ we found much smaller occupation numbers and "squeezed" resonance bands.) Oscillations with amplitude $\sigma_{0} \geq v$ will certainly not be harmonic, see e.g. 12]. However, this is only a schematic and qualitative example anyway, since it completely neglects the nonlinearities in the $\pi$-field and the back-reaction on the $\sigma$, which are most essential in the lattice simulation [13]. We thus do not attempt to be more "realistic" in this respect.

The strong amplification of the soft modes of the $\pi$ field, $k \sim 0$ has been interpreted as being due to an imaginary effective mass of the pion [7],8]. However, there are additional resonance bands at higher $k$, being particularly pronounced if the amplitude of the $\sigma$-field is big.

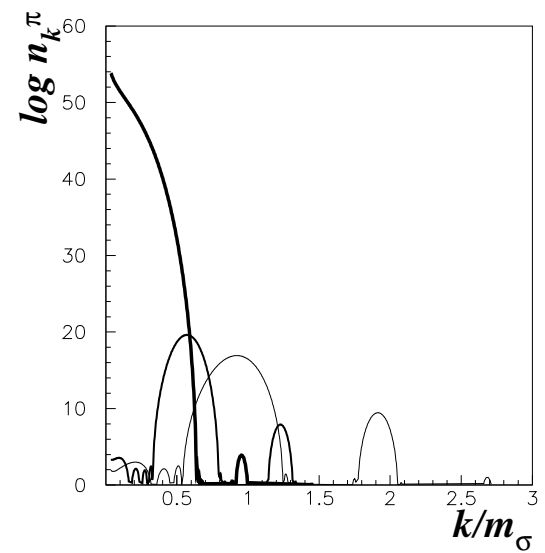

FIG. 5. Occupation numbers of various Fourier modes of the $\pi$-field after 10 oscillations of the $\sigma$-field with amplitude $\sigma_{0}=v$ (thick line with very strong soft modes), $\sigma_{0}=2 v$ (thinner line), and $\sigma_{0}=m_{\sigma}$ (thinnest line), respectively.

In Fig. 6 we show the energy density of the $\pi$-field,

$$
e^{\pi}=3 \int \frac{d^{3} k}{(2 \pi)^{3}} \Omega_{k} n_{k}^{\pi} .
$$

The factor 3 in front of the integral comes from the threefold isospin degeneracy of the $\pi$-field. This is the energy density that would be measured if the classical field decayed into particles with energy $\Omega_{k}$ at the corresponding time. One observes that the energy density of the $\pi$-field increases exponentially in time. In a more realistic calculation, as e.g. the lattice simulation described above, that exponential growth will shut off eventually due to energy conservation and the back-reaction.

In a case like this we do not integrate out the $k>0$ modes, or the modes above some cutoff $k_{c} \ll T$, and treat only the zero-mode classically [17] (evolving in the effective potential generated by the "hard" modes above $\left.k_{c}\right)$. In particular, we can not compute the dissipative corrections to the classical dynamics of the soft modes, which is due to scattering with the "hard" modes, perturbartively (for an alternative approach see [18]). We therefore decided to describe all modes within the first few parametric resonance bands classically and to solve the full nonlinear field equations (on the lattice). 
Of course, we can not include arbitrarily hard modes in our classical description as we would run into the wellknown Rayleigh-Jeans type of ultraviolet divergences, cf. e.g. the discussion in [17]. Fortunately, our lattice simulations were practically independent of the lattice spacing (domains always formed) as long as $a \simeq 0.1-0.3 \mathrm{fm}$, showing that higher resonance bands do not contribute to the classical evolution. One may have to employ smaller $a$ (i.e. larger $k_{c}$ ) if the initial fluctuations were substantially larger than 0.3 .

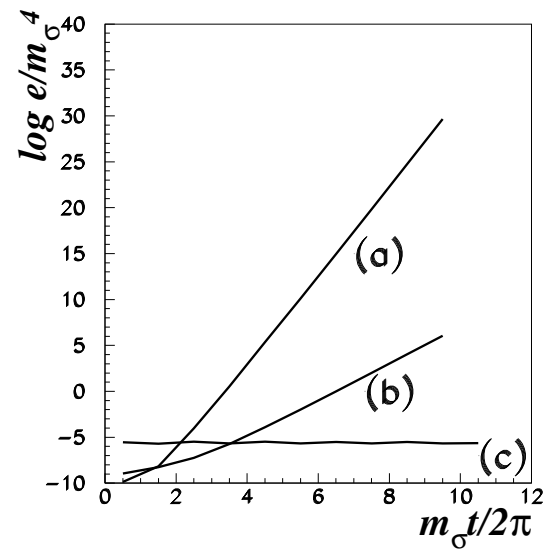

FIG. 6. Energy density of the $\pi$-field (exponentially increasing) and of the quarks (constant; only modes up to $k=6 m_{\sigma}$ ), averaged over one period of oscillation of the $\sigma$-field. $\sigma_{0}=v$ for curves (a,c), $\sigma_{0}=2 v$ for curve (b).

Furthermore, we found that the $\sigma$-field relaxes to a value more closely to $f_{\pi}$, the correct vacuum value, if $a$ is small. We obtained $\langle\sigma\rangle=0.80 f_{\pi}$ for $a=0.2 \mathrm{fm}$ while $\langle\sigma\rangle=0.56 f_{\pi}$ for $a=1 \mathrm{fm}$; both values determined at $t=120 \mathrm{fm}$; see Fig. 8. This observation is opposite to the expectation in the static, thermal equilibrium case [17]. In the dynamical case the high-frequency classical fluctuations, which would otherwise mimic finite-temperature contributions to the potential, cool more efficiently by transfering more of their energy to the soft modes if $a$ is small (as is obvious from Fig. 2 and 3). This is not in contradiction to the fact that for $t \rightarrow \infty$ one should have equipartitioned energy (classically) as the reverse process, energy transfer from soft to hard modes, occurs on much longer time-scales. Thus, we employ $k_{c} \simeq 1.5 m_{\sigma}$ in the simulation of the model on the lattice (described above). In this way, we account for the full non-linear evolution of those parametrically amplified modes.

The modes above $k_{c}$ could be accounted for by integrating them out of the partition function, assuming e.g. a thermal distribution [9, 17]. The scattering of modes with $k \leq k_{c}$ off modes with $k>k_{c}$ will then introduce fluctuation and dissipation terms into the classical equations of motion. Alternatively, the interactions between classical modes and quantum fluctuations could even be treated explicitly, such that the latter effectively act as a heat-bath for the former 18 . That is, however, beyond the scope of the present manuscript.

We now reintroduce the coupling of the $\sigma$-field to the quarks. For the constituent quark mass $m_{q}=g \sqrt{\sigma^{2}+\pi^{2}}$ in the vacuum to be one third of the proton mass, we employ $g=3.3$. Furthermore, dropping the $\pi$-field and again assuming a homogeneous $\sigma$-field, one arrives at the following equation of motion for modes of the auxilliary field $\psi 19$,

$$
\ddot{\psi}_{k}+\left\{k^{2}+g^{2}(\sigma+v)^{2}-i g \dot{\sigma}\right\} \psi_{k}=0,
$$

where $\sigma=\sigma_{0} \sin m_{\sigma} t$, as above. The quark wavefunction can be obtained from $\psi_{k}$ as

$$
\begin{aligned}
q(\vec{x}, t)= & \sum_{r}[i \gamma \cdot \partial+g \sigma] \int \frac{d^{3} k}{(2 \pi)^{3} 2 \Omega_{k}(t=0)} \\
& \times b_{\vec{k}, r} e^{i \vec{k} \cdot \vec{x}} \psi_{k}(t) R_{r} .
\end{aligned}
$$

$R_{r}$ denote the two eigenvectors of $\gamma^{0}$ with eigenvalue +1 . Again, the real part of the expression in curly brackets in eq. (0.7) denotes the oscillator frequency $\Omega_{k}^{2}$. As initial condition we employ

$$
\psi_{k}(t=0)=\mathcal{N}_{k} \quad, \quad \dot{\psi}_{k}(t=0)=-i \Omega_{k} \mathcal{N}_{k} \quad,
$$

with $1 / \mathcal{N}_{k}=\sqrt{2 \Omega_{k}\left(\Omega_{k}+g \sigma+g v\right)}$. The occupation number of each mode is

$$
n_{k}=\frac{1}{2}-\frac{k^{2}}{\Omega_{k}} \operatorname{Im}\left(\psi_{k} \dot{\psi}_{k}^{*}\right)-g \frac{\sigma+v}{2 \Omega_{k}},
$$

and the energy density is given by expression (0.6), with a factor 24 (instead of 3 ) in front of the integral.

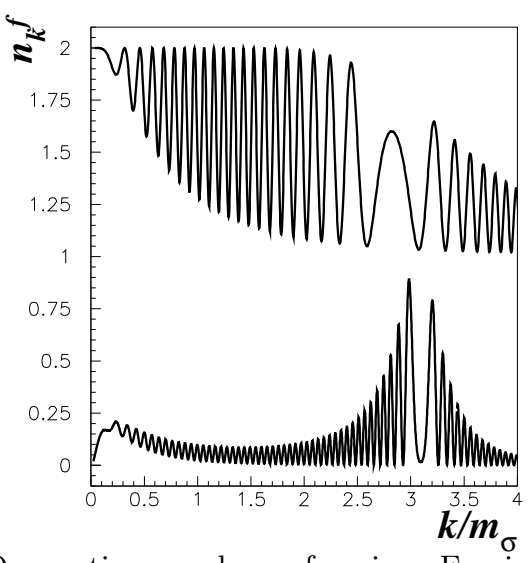

FIG. 7. Occupation numbers of various Fourier modes of the $\psi$-field after 10 oscillations of the $\sigma$-field with amplitude $\sigma_{0}=v$ (bottom curve) and $\sigma_{0}=m_{\sigma}$ (top curve; $1+n_{k}^{f}$ is shown in that case). 
Fig. 7 shows the occupation number of the various fermion modes. Due to the Pauli principle exponential growth of the occupation numbers is not possible. However, while the incoherent process $\sigma \rightarrow q \bar{q}$ with "on-shell" kinematics is forbidden (the quark mass, averaged over one oscillation of the $\sigma$-field, is $m_{q}=g\left(f_{\pi}+2 \sigma_{0} / \pi\right)>$ $\left.m_{\sigma} / 2\right)$, the $\sigma$-modes can act as coherent field to produce fermions. This is because the quark-mass becomes small during some time-interval of the coherent $\sigma$-oscillations and the frequency $\Omega_{k}$ of the quark wavefunction can drop below $m_{\sigma}$ (one can show analytically that no quarks are being produced if the $\sigma$-field is static relative to $\psi_{k}$ ). As opposed to the bosonic occupation numbers, small- $k$ quarks are produced if the amplitude of the driving $\sigma$ field is large. However, even in this very simple analysis disrespecting total energy conservation the long-time energy density of the quarks is well below that of the pions. Only if the $\sigma$-oscillations were strongly damped, e.g. by a rapidly expanding background, quark-pair production may be as important as pion production. The discussion of the condensate relaxation in an expanding metric is out of the scope of the present manuscript, see e.g. [6.,7.

In summary, we have performed numerical simulations of the classical dynamical evolution of the chiral condensates in strong interaction on a lattice. In particular, we have focused on the situation where the chiral fields are rapidly quenched from an initial state with fluctuations.

We confirmed previous results in that the spinodal decomposition process does not lead to formation of big domains of coherent $\pi$-field. This remains true also at later times if indeed only the zero-mode can be described classically, i.e. for rather large lattice spacing $a \geq 1 \mathrm{fm}$. However, if higher modes are also treated classically by decreasing the lattice spacing to $\simeq 0.1-0.2 \mathrm{fm}$, longrange correlations (domains) do form as a consequence of parametric resonance and the nonlinear dynamics of those exponentially amplified modes. Despite evident limitations of the effective model that we employed, we think that the strong amplification of "hard" modes of the condensates, which as a consequence evolve nonlinearly and eventually drive the amplification of the "soft" modes, may occur also in more realistic models.

Finally, we have studied very briefly the effect of coupling the chiral fields to constituent quarks. Despite the fact that the Dirac vacuum is not stable in the presence of coherent classical $\sigma$-field oscillations with big amplitude or high frequency, the energy density of the quarks remains well below that of the $\pi$-field modes at late time. Thus, the coupling of the condensates to quarks is not likely to destroy the "pseudoscalar laser" emerging if an initial state with fluctuations is rapidly "quenched" to the spontaneously broken chiral symmetry.

\section{ACKNOWLEDGMENTS}

We are happy to thank C. Greiner, M. Gyulassy, A. Jackson, L. McLerran, I. Mishustin, R. Pisarski, D. Rischke, and D. Son for fruitful discussions and useful comments; and L. McLerran for initiating this study. A.D. acknowledges support from the DOE Research Grant, Contract No. De-FG-02-93ER-40764.

[1] J.D. Bjorken, Int. J. Mod. Phys. A7, 4189 (1992).

[2] A.A. Anselm, Phys. Lett. B217, 169 (1989); A.A. Anselm and M.G. Ryskin, Phys. Lett. B266, 482 (1991); L.P. Csernai and I.N. Mishustin, Phys. Rev. Lett. 74, 5005 (1995); A. Abada and M. Birse, Phys. Rev. D55, 6887 (1997); T.S. Biro, D. Molnar, Z. Feng and L.P. Csernai, Phys. Rev. D55, 6900 (1997).

[3] K. Rajagopal and F. Wilczek, Nucl. Phys. B399, 395 (1993); Nucl. Phys. B404, 577 (1993).

[4] S. Gavin, A. Gocksch and R.D. Pisarski, Phys. Rev. Lett. 72, 2143 (1994).

[5] D. Boyanovsky, H.J. de Vega and R. Holman, Phys. Rev. D51, 734 (1995).

[6] O. Scavenius and A. Dumitru, Phys. Rev. Lett. 83, 4697 (1999).

[7] M. Asakawa, Z. Huang and X. Wang, Phys. Rev. Lett. 74, 3126 (1995); J. Randrup, Phys. Rev. Lett. 77, 1226 (1996); T.S. Biro and C. Greiner, Phys. Rev. Lett. 79, 3138 (1997).

[8] J.P. Blaizot and A. Krzywicki, Phys. Rev. D46, 246 (1992); and Acta Phys. Polon. 27, 1687 (1996);

[9] D. Boyanovsky, D. Lee and A. Singh, Phys. Rev. D48, 800 (1993).

[10] L. Kofman, A. Linde and A.A. Starobinsky, Phys. Rev. Lett. 73, 3195 (1994); Phys. Rev. D56, 3258 (1997).

[11] H. Hiro-Oka and H. Minakata, Phys. Rev. C61, 044903 (2000).

[12] D.I. Kaiser, Phys. Rev. D59, 117901 (1999).

[13] S.Y. Khlebnikov and I.I. Tkachev, Phys. Rev. Lett. 77, 219 (1996).

[14] R.D. Pisarski and F. Wilczek, Phys. Rev. D29, 338 (1984).

[15] M. Gell-Mann and M. Levy, Nuovo Cim. 16, 705 (1960).

[16] W.H. Press, S.A. Teukolsky, W.T. Vetterling and B.P. Flannery, "Numerical Recipes in C", 2.ed., Cambridge University Press 1992.

[17] D. Bödeker, L. McLerran and A. Smilga, Phys. Rev. D52, 4675 (1995); S. Jeon, Phys. Rev. D52, 3591 (1995); C. Greiner and B. Müller, Phys. Rev. D55, 1026 (1997); D.H. Rischke, Phys. Rev. C58, 2331 (1998).

[18] F. Cooper, S. Habib, Y. Kluger and E. Mottola, Phys. Rev. D55, 6471 (1997).

[19] P.B. Greene and L. Kofman, Phys. Lett. B448, 6 (1999). 


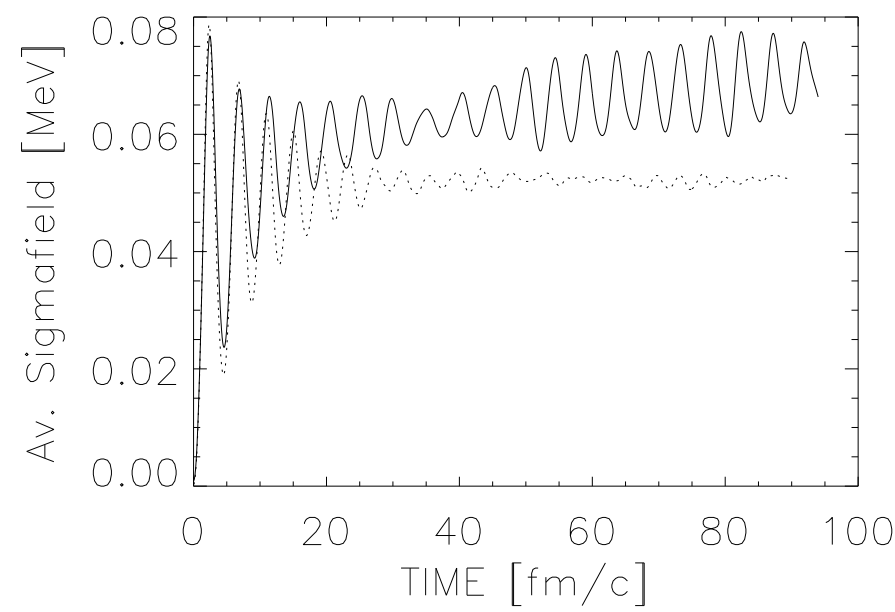

FIG. 8. Additional Figure: $\sigma$-field (averaged over the entire lattice) as a function of time for $a=0.2 \mathrm{fm}$ (full line) and $a=1 \mathrm{fm}$ (dotted line). 